OPEN ACCESS

Edited by:

Liana Gheorghe,

Fundeni Clinical Institute, Romania

Reviewed by:

Winnie S. S. Chee,

International Medical

University, Malaysia

Arthur Orieux

Centre Hospitalier Universitaire de

Bordeaux, France

*Correspondence:

Honghong $\mathrm{Pe}$

peihhiz@163.com

†These authors have contributed equally to this work

Specialty section:

This article was submitted to Clinical Nutrition,

a section of the journal

Frontiers in Nutrition

Received: 31 May 2021

Accepted: 30 July 2021

Published: 25 August 2021

Citation:

Lv J, Wang H, Sun B, Gao Y, Zhang Z and Pei $H$ (2021) Serum Albumin

Before CRRT Was Associated With

the 28- and 90-Day Mortality of

Critically III Patients With Acute Kidney

Injury and Treated With Continuous

Renal Replacement Therapy.

Front. Nutr. 8:717918

doi: 10.3389/fnut.2021.717918

\section{Serum Albumin Before CRRT Was Associated With the 28- and 90-Day Mortality of Critically III Patients With Acute Kidney Injury and Treated With Continuous Renal Replacement Therapy}

\author{
Junhua Lv ${ }^{1+}$, Hai Wang ${ }^{2 \dagger}$, Baoni Sun ${ }^{1}$, Yanxia Gao ${ }^{1}$, Zhenglinag Zhang ${ }^{1}$ and \\ Honghong Pei ${ }^{1 *}$
}

'Emergency Department, The Second Affiliated Hospital of Xi'an Jiaotong University, Xi'an, China, ${ }^{2}$ Department of Hepatobiliary Surgery, The First Affiliated Hospital of Xi'an Jiaotong University, Xi'an, China

Introduction: Although low serum albumin (ALB) may worsen acute kidney injury (AKI), additional study is needed to establish the connection between ALB and the prognosis of critically ill patients with $\mathrm{AKI}$ and treated with continuous renal replacement therapy (CRRT).

Methods: A secondary analysis of a bi-center, retrospective, and observational study, such as critically ill patients with AKI and treated with CRRT from January 2009 to September 2016. The univariate analysis, multi-factor regression analysis, sensitivity analysis, and curve-fitting analysis were applied to explore the association of ALB with the 28 and 90 days mortality of critically ill patients with AKI and treated with CRRT, and the removal efficiency of serum phosphorus.

Results: From January 2009 to September 2016, 1,132 cases with AKI and treated with CRRT met the inclusion criteria and enrolled in this study. We found that the higher ALB before CRRT, the lower the 28- and 90-day mortality of patients with AKI and treated with CRRT, the higher removal efficiency of serum phosphorus, the adjusted hazard ratio $(\mathrm{HR})$ value for 28-day mortality in the four models were separately $0.92(0.90,0.95)$, 0.91 (0.89, 0.94), $0.92(0.89,0.95)$, and $0.92(0.89,0.95)$; the adjusted HR value for 90 day mortality in the four models were $0.91(0.89,0.94), 0.92(0.89,0.95), 0.92(0.89$, $0.95)$, and $0.92(0.89,0.96)$; the adjusted OR value for the removal efficiency of serum phosphorus in the four models were separately $-0.04(-0.07,-0.01),-0.05(-0.08$, $-0.01),-0.04(-0.08,-0.01)$, and $-0.04(-0.08,-0.01)$. The sensitivity analysis and curve-fitting analysis also showed that ALB before CRRT was correlated with the 28 and 90 days mortality of critically ill patients with $\mathrm{AKI}$ and treated with CRRT and the removal efficiency of serum phosphorus. 
Conclusion: The higher the serum ALB before CRRT, the lower the mortality of critically ill patients with $\mathrm{AKI}$ and treated with CRRT, and the higher the clearance efficiency of serum phosphorus.

Keywords: serum albumin, acute kidney infusion, 28- and 90-day mortality, critically ill patients, continuous renal replacement therapy

\section{INTRODUCTION}

Acute kidney injury (AKI) is a frequent complication of critically ill patients. Approximately $30-50 \%$ of critically ill patients develop AKI, and the mortality for individuals with AKI may reach $50 \%$ (1). Approximately $40 \%$ of the AKI patients may advance to life-threatening renal dysfunction $(1,2)$, such as hyperkalemia, severe acidosis, severe azotemia, oliguria, or continuous anuria, and will need renal replacement treatment, among which continuous renal replacement therapy (CRRT) is the most commonly used renal replacement therapy in the intensive care unit (ICU) (1). The efficiency of CRRT clearance is closely related to the prognosis of patients (3). According to various research studies, the serum phosphorus clearance of CRRT is linked to the serum creatinine and urea nitrogen clearance (4-6). Simultaneously, serum phosphorus clearance of CRRT is linked to the prognosis of critically ill patients $(7,8)$. ALB is one of the most critical proteins in the human plasma because it may maintain plasma colloid osmotic pressure, engage in material transport in blood circulation, and facilitate communication among the intracellular fluid, extracellular fluid, and tissue fluid (9). Hypoalbuminemia is widespread in patients with critical illnesses, and it is widely recognized as being linked to patient deterioration and higher death $(10,11)$. The incidence of hypoproteinemia in hospitalized patients is $\sim 21 \%$ (12), but its incidence in acute illness is more than 50\% (13).

In addition, ALB is closely related to the occurrence and progression of AKI. According to David R Williamson's study, ALB administration was linked with a dose-dependent risk of AKI associated with colloids after heart surgery (14). Low serum ALB was shown to be an independent risk factor for AKI in the meta-analysis of Michael Joannidis, which comprised 43 retrospective observational cohort studies that include 68,000 patients (15).

However, fewer studies have been conducted to investigate the relationship between serum ALB and the prognosis of critically ill patients with AKI and treated with CRRT, and it is unclear whether serum ALB affects the clearance efficiency of serum phosphorus. Therefore, this research assumes that the higher the serum ALB before CRRT, the lower the mortality of severe AKI patients and the higher the serum phosphorus clearance efficiency.

Abbreviations: CRRT, continuous renal replacement therapy; ALB, albumin; AKI, acute kidney injury; AKIN, acute kidney injury network; CKD, chronic kidney disease; ICU, intensive care unit; BMI, body mass index; MAP, mean arterial pressure; CCI score, Charlson comorbidity index; COPD. chronic obstructive pulmonary disease; $\mathrm{WBC}$, white blood cell; $\mathrm{HB}$, hemoglobin; BUN, blood urea nitrogen; $\mathrm{Cr}$, creatinine; CRP, C-reactive protein; SOFA, sequential organ failure assessment; $\mathrm{CVVH}$, continuous venovenous Hemofiltration.

\section{METHODS \\ Study Design}

A secondary analysis of a bi-center, retrospective, and observational study including critically ill patients with acute kidney injury (AKI) and treated with CRRT from January 2009 to September 2016 at Yonsei University Health System Severance Hospital and National Health Insurance Service Medical Center Ilsan hospital, Republic of Korea.

\section{Objective}

The study aimed to explore the relationship of ALB with the 28and 90-day mortality of patients with AKI and treated with CRRT and with the removal efficiency of serum phosphorus.

\section{Ethics Approval and Consent to Participate}

New ethics permission and consent to participate were not applicable since the original author had received ethical approval while performing this research, and our study was a retrospective analysis of data reuse.

\section{Data Source}

The data used in this study were shared by Seung Hyeok Han, which were stored in the Dryad database (https://datadryad.org// resource/doi:10.5061/dryad.6v0j9) (7). The database is a public data repository, which contains data uploaded by the authors to make their research data discoverable, freely reusable, and citable.

\section{Inclusion Criteria}

(1) Patients were complicated with AKI and treated with CRRT in the intensive care unit (ICU); (2) the stage of AKI was two or more according to the acute kidney injury network (AKIN) criteria, in which serum creatinine and urine outputs were taken into account.

\section{Exclusion Criteria}

Patients with the following situation were excluded in this study: (1) age < 18 years; (2) pregnant or lactating women; (3) with postrenal obstruction; (4) with Stage 5 chronic kidney disease (CKD), kidney transplantation, dialysis, or CRRT; (5) the value of ALB was missing or outliers.

\section{Participants}

From January 2009 to September 2016, 2,110 patients were presented with AKIN stage 2 or more and treated with CRRT, of which 978 patients were excluded in the ICU at Yonsei University Health System Severance Hospital and National Health Insurance Service Medical Center Ilsan hospital: (1) age $<18$ years $(n=42)$; (2) pregnant or lactating women $(n=12)$; (3) with postrenal obstruction $(n=263)$; (4) with the history of 
stage 5 CKD, kidney transplantation, dialysis, or CRRT $(n=585)$; (5) the value of ALB was missing $(n=9)$ or outliers $(n=3)$, the ALB of two patients were $0 \mathrm{~g} / \mathrm{L}$, and the another was $5.9 \mathrm{~g} / \mathrm{L}$ ). Finally, a total of 1,132 patients with AKI and treated with CRRT were included in the current study (as shown in Figure 1).

\section{Grouping}

According to ALB before CRRT, patients were divided into three groups: $\mathrm{ALB}<25 \mathrm{~g} / \mathrm{L}(n=436), 25 \mathrm{~g} / \mathrm{L} \leq \mathrm{ALB}<30 \mathrm{~g} / \mathrm{L}$ $(n=401)$, and $30 \mathrm{~g} / \mathrm{L} \leq \mathrm{ALB}$.

\section{The Outcome Indicators}

(1) 28 and 90 days mortality; (2) the removal efficiency of serum phosphorus, delta phosphate $=$ phosphate $(24 \mathrm{~h})$ phosphate $(0 \mathrm{~h})$.

\section{Collection of Clinical and Biochemical Data}

The following variables were included in the current study, such as age, sex, body mass index (BMI), mean arterial pressure (MAP), complications (myocardial infarction, congestive heart failure, cerebrovascular disease, peripheral vascular disease, dementia, diabetes mellitus, hypertension, and chronic obstructive pulmonary disease), Charlson comorbidity index (CCI) score, biochemical laboratory tests, e.g., $\mathrm{K}^{+}, \mathrm{HCO}^{-}$, phosphate $(0 \mathrm{~h})$, phosphate $(24 \mathrm{~h})$, delta phosphate, white blood cell (WBC), hemoglobin (HB), blood urea nitrogen (BUN), creatinine $(\mathrm{Cr})$ and $\mathrm{C}$-reactive protein $(\mathrm{CRP})$, and sequential organ failure assessment (SOFA) score. Age, gender, BMI, and CCI scores were collected at the time of admission; and the other variables were collected at $0 \mathrm{~h}$ of CRRT.

\section{CRRT Protocol}

The nephrologist assessed whether the patients should be treated with CRRT based on the development of AKI in the ICU patients. The CRRT machines were the multiFiltrate (Fresenius Medical Care, Bad Homburg, Germany) or the Prismaflex (Baxter International Inc. Lundia $\mathrm{AB}$, Sweden). The applied dialyzers had a surface area of 1.0-1.4 $\mathrm{m}^{2}$ with a sieving coefficient for albumin and 32 -microglobulin of 0.001 and $0.58-0.65$, respectively. The parameters of CRRT were the following: (1) model: Continuous venovenous hemofiltration $(\mathrm{CVVH})$ through the internal jugular, subclavian, or femoral vein; (2) blood flow: the start was at $100 \mathrm{ml} / \mathrm{min}$ and up to $150 \mathrm{ml} / \mathrm{min}$; (3) The total dialysis and replacement dose were targeted to deliver $\geq 35 \mathrm{ml} / \mathrm{kg} / \mathrm{h}$ in all the patients.

\section{Statistical Analysis}

(1) Statistical description: Mean $\pm \mathrm{SD}$ ( $\mathrm{x} \pm \mathrm{s}$ ) was used for the continuous variables of baseline data in the groups, and counts numerical values and percentages were shown in the data. The data were compared using the $t$-test, if continuous data had a normal distribution and homogeneity of variance. Mann-Whitney's $U$-test was performed if the continuous data did not meet the normal distribution or homogeneity of the variance. For categorical data, the $\chi^{2}$-test was utilized. (2) Analyze the relationship between ALB and 28- and 90day mortality of patients, and delta phosphate by univariate and multivariate analysis. A multivariate Cox regression was performed for 28- and 90-day mortality, and multivariate logistic regression was performed for delta phosphate. (3) To further understand the relationship between ALB and 28- and 90-day mortality of patients, subgroup analyses were performed on the age, MAP, congestive heart failure, hypertension, AKIN stage,

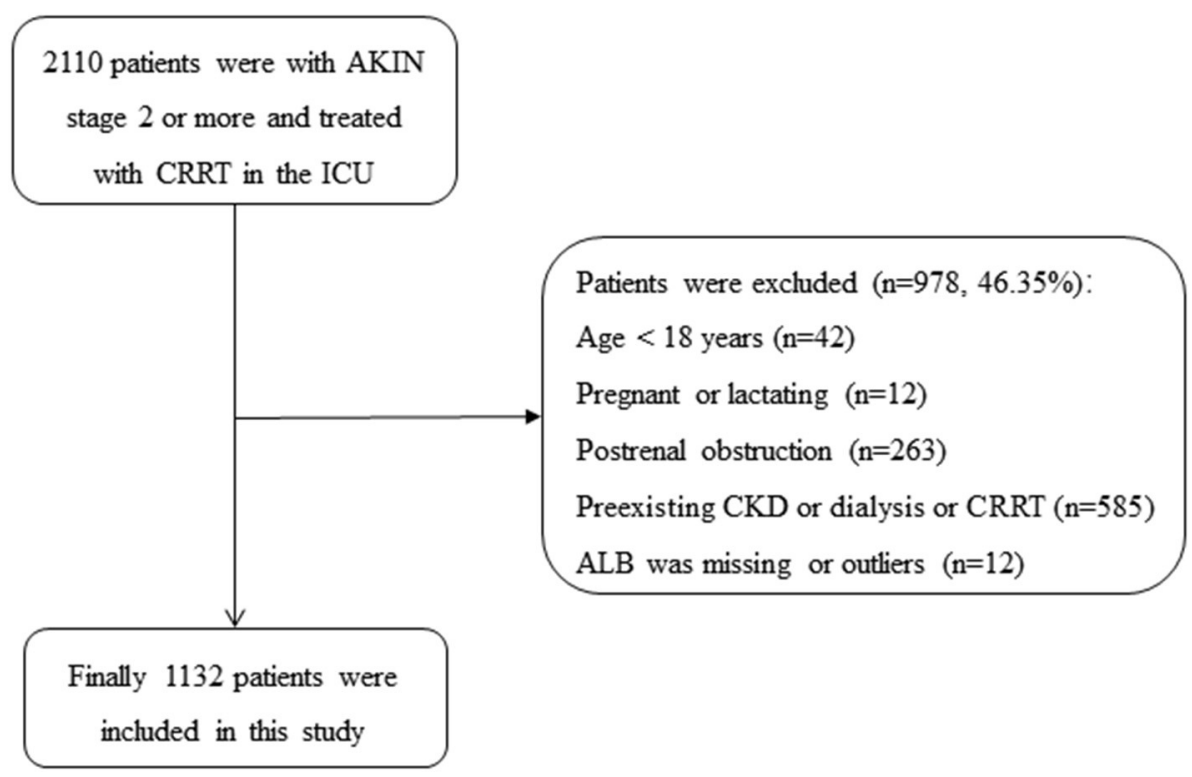

FIGURE 1 | Flowchart of patient selection. AKIN, acute kidney injury network; ALB, albumin; CKD, chronic kidney diseases; CRRT, continuous renal replacement therapy; ICU, intensive care unit. 
mechanical ventilation, SOFA score, AKI causes, and CRRT causes. (4) The selection of adjustment variables were by the following: if the confounders influenced the effective estimate of ALB by more than $10 \%$ and identified with the literature, we would adjust it. (5) Curve fitting analysis was used to investigate the connection among ALB, 28- and 90-day mortality, and delta phosphate. All statistical analyses were carried out by EmpowerStats 2.0 (Copyright $2009 \mathrm{X} \& \mathrm{Y}$ Solutions, Inc.) and $\mathrm{R}$ software (version 3.4.3). The value of $P<0.05$ was statistically significant.

TABLE 1 | The clinical characteristics of patients.

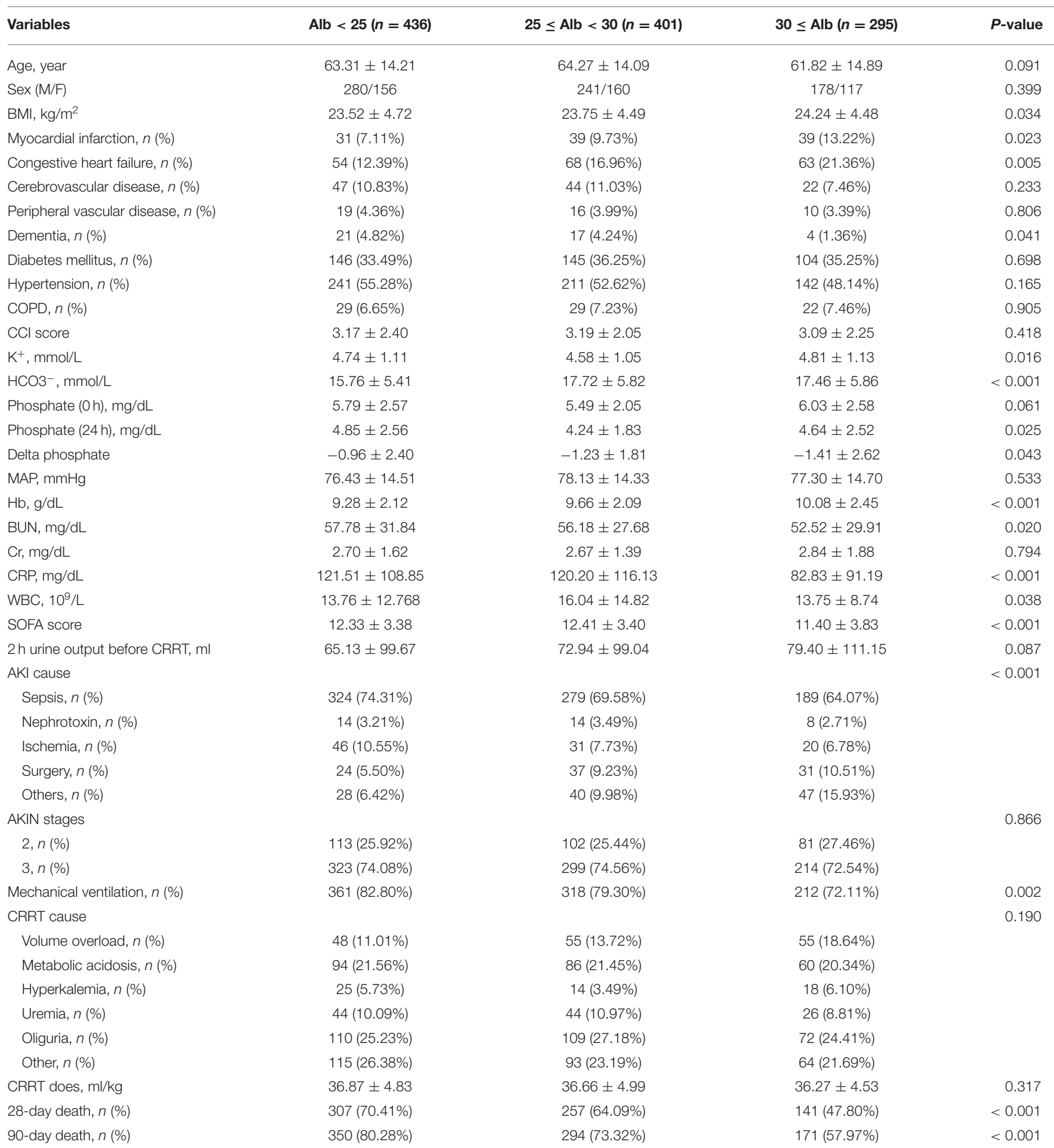




\section{RESULTS}

\section{Baseline Characteristics}

In this research, 1,132 patients with AKI and treated with CRRT were included. The ages of three groups included were as follows: ALB $<25 \mathrm{~g} / \mathrm{L}(n=436), 25 \mathrm{~g} / \mathrm{L} \leq \mathrm{ALB}<30 \mathrm{~g} / \mathrm{L}(n=401)$, and $30 \mathrm{~g} / \mathrm{L} \leq \mathrm{ALB}$ were $63.31 \pm 14.21,64.27 \pm 14.09$, and $61.82 \pm 14.89$ years, respectively, with no significant difference $(P=0.091)$. The male-to-female ratio among the three groups was 280/156 (1.79), 241/160 (1.51), and 178/117 (1.52), with no statistical difference $(P=0.399)$. The BMI among the three groups was $23.52 \pm 4.72,23.75 \pm 4.49$, and $24.24 \pm 4.48$ $\mathrm{kg} / \mathrm{m}^{2}$, respectively, with statistical significance $(P=0.034)$. The 28-day mortality among the three groups was 307 (70.41\%), $257(64.09 \%)$, and $141(47.80 \%)$, respectively, with statistical significance $(P<0.001)$. The 90 -day mortality among the three groups was $350(80.28 \%), 294(73.32 \%)$, and $171(57.97 \%)$, respectively, with statistical significance $(P<0.001)$. There was a significant difference between myocardial infarction, congestive heart failure, and dementia $(P<0.05)$. There was a significant difference in $\mathrm{HCO}^{3-}$, phosphate $(24 \mathrm{~h})$, delta phosphate, $\mathrm{HB}$, CRP, WBC, SOFA score, AKI cause, and CRRT cause across the three groups $(P<0.05)$. There was no significant difference in the other variables between the groups $(P>0.05)$ (as shown in Table 1).

\section{ALB Was Associated With the 28- and 90-day Mortality of Patients With AKI and Treated With CRRT}

When ALB was used as a continuous variable, the higher the serum ALB before CRRT, the lower the mortality of critically ill patients with AKI and treated with CRRT. The adjusted hazard ratio (HR) value for 28-day mortality in the four models were separately $0.92(0.90,0.95), 0.91(0.89,0.94), 0.92(0.89,0.95)$, and $0.92(0.89,0.95)$. The adjusted HR value for 90 -day mortality in the four models were $0.91(0.89,0.94), 0.92(0.89,0.95), 0.92(0.89$, $0.95)$, and $0.92(0.89,0.96)$. When ALB was used as a classification variable and $\mathrm{ALB}<25 \mathrm{~g} / \mathrm{L}$ as a reference, it was discovered that when $25 \mathrm{~g} / \mathrm{L} \leq \mathrm{ALB}<30 \mathrm{~g} / \mathrm{L}$ and $30 \mathrm{~g} / \mathrm{L} \leq \mathrm{ALB}$, the 28- and 90day mortality of patients with AKI and treated with CRRT were substantially decreased (as shown in Table 2).

\section{The Sensitivity Analysis Was Used to Detect the Relationship Between the ALB and the 28- and 90-day Mortality of Patients With AKI and Treated With CRRT}

Age, MAP, myocardial infarction, congestive heart failure, diabetes mellitus, hypertension, AKIN stage, mechanical ventilation, SOFA score, AKI causes, and CRRT causes were

TABLE 2 | Multivariate logistic regression analysis for 28- and 90-day mortality.

\begin{tabular}{|c|c|c|c|c|}
\hline Exposure & 28-day mortality (Adjusted HR 95\% Cl) & $P$-value & 90-day mortality (Adjusted HR 95\% Cl) & $P$-value \\
\hline \multicolumn{5}{|l|}{ Model 1} \\
\hline Alb, g/L & $0.92(0.90,0.95)$ & $<0.001$ & $0.91(0.89,0.94)$ & $<0.001$ \\
\hline \multicolumn{5}{|l|}{ Alb, g/L } \\
\hline$<25$ & 1.00 (Reference) & & 1.00 (Reference) & \\
\hline $25 \leq$ and $<30$ & $0.71(0.53,0.96)$ & 0.027 & $0.62(0.45,0.87)$ & 0.006 \\
\hline $30 \leq$ & $0.37(0.27,0.51)$ & $<0.001$ & $0.32(0.23,0.45)$ & $<0.001$ \\
\hline \multicolumn{5}{|l|}{ Model 2} \\
\hline Alb, g/L & $0.91(0.89,0.94)$ & $<0.001$ & $0.92(0.89,0.95)$ & $<0.001$ \\
\hline \multicolumn{5}{|l|}{ Alb, g/L } \\
\hline$<25$ & 1.00 (Reference) & & 1.00 (Reference) & \\
\hline $25 \leq$ and $<30$ & $0.54(0.36,0.80)$ & 0.002 & $0.53(0.35,0.81)$ & 0.003 \\
\hline $30 \leq$ & $0.35(0.23,0.54)$ & $<0.001$ & $0.37(0.24,0.59)$ & $<0.001$ \\
\hline \multicolumn{5}{|l|}{ Model 3} \\
\hline Alb, g/L & $0.92(0.89,0.95)$ & $<0.001$ & $0.92(0.89,0.95)$ & $<0.001$ \\
\hline \multicolumn{5}{|l|}{ Alb, $g / L$} \\
\hline$<25$ & 1.00 (Reference) & & 1.00 (Reference) & \\
\hline $25 \leq$ and $<30$ & $0.57(0.38,0.87)$ & 0.009 & $0.56(0.36,0.88)$ & 0.011 \\
\hline $30 \leq$ & $0.35(0.22,0.56)$ & $<0.001$ & $0.39(0.24,0.63)$ & $<0.001$ \\
\hline \multicolumn{5}{|l|}{ Model 4} \\
\hline Alb, g/L & $0.92(0.89,0.95)$ & $<0.001$ & $0.92(0.89,0.96)$ & $<0.001$ \\
\hline \multicolumn{5}{|l|}{ Alb, g/L } \\
\hline$<25$ & 1.00 (Reference) & & 1.00 (Reference) & \\
\hline $25 \leq$ and $<30$ & $0.58(0.37,0.91)$ & 0.017 & $0.59(0.37,0.94)$ & 0.027 \\
\hline $30 \leq$ & $0.37(0.22,0.60)$ & $<0.001$ & $0.41(0.24,0.68)$ & $<0.001$ \\
\hline
\end{tabular}

Model 1: Adjusted for age; sex; body mass index (BMI); Charlson comorbidity index (CCl score). Model 2: Adjusted for model 1 plus C-reactive protein (CRP), white blood cell (WBC),

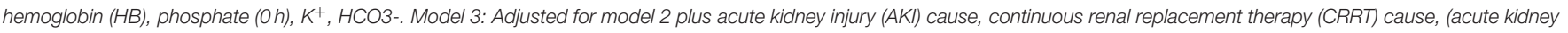
injury network (AKIN) stages, CRRT does, 2 h urine output before CRRT initiation. Model 4: Adjusted for model 3 plus sequential organ failure assessment (SOFA) score. 
all subjected to sensitivity analysis. ALB was associated with 28-day mortality in the sensitivity analysis, except in individuals with myocardial infarction, AKIN stage 2, or CRRT cause (hyperkalemia, uremia, and oliguria). Additional sensitivity analysis for 90-day mortality revealed that ALB was linked with 90-day death in all the patients except those with myocardial infarction, congestive heart failure, AKIN stage 2, or CRRT cause (hyperkalemia, uremia, and oliguria; as shown in Table 3).

\section{ALB Was Associated With the Removal Efficiency of Phosphate}

When ALB was used as a continuous variable, the higher the serum ALB before CRRT treatment, the higher the clearance efficiency of serum phosphorus. The adjusted OR values for delta phosphate in the four models were $-0.04(-0.07,-0.01)$, $-0.05(-0.08,-0.01),-0.04(-0.08,-0.01)$, and -0.04 $(-0.08,-0.01)$. When ALB was used as a classification variable, it

TABLE 3 | The subgroup analysis of multivariate logistic regression analysis for 28 - and 90-day mortality.

\begin{tabular}{|c|c|c|c|c|}
\hline Exposure & 28-day mortality (Adjusted HR 95\% CI) & $P$-value & 90-day mortality (Adjusted HR 95\% Cl) & $P$-value \\
\hline \multicolumn{5}{|l|}{ Age, year } \\
\hline$<65$ & $0.95(0.92,0.98)$ & 0.001 & $0.97(0.94,1.00)$ & 0.031 \\
\hline $65 \leq$ & $0.96(0.93,0.98)$ & $<0.001$ & $0.94(0.92,0.97)$ & $<0.001$ \\
\hline \multicolumn{5}{|l|}{ MAP, mmHg } \\
\hline$<65$ & $0.91(0.87,0.95)$ & $<0.001$ & $0.94(0.91,0.98)$ & 0.006 \\
\hline $65 \leq$ & $0.96(0.94,0.98)$ & $<0.001$ & $0.96(0.94,0.98)$ & $<0.001$ \\
\hline \multicolumn{5}{|l|}{ Myocardial infarction } \\
\hline Yes & $1.05(0.95,1.17)$ & 0.315 & $0.98(0.88,1.08)$ & 0.638 \\
\hline No & $0.96(0.94,0.98)$ & $<0.001$ & $0.96(0.94,0.98)$ & $<0.001$ \\
\hline \multicolumn{5}{|c|}{ Congestive heart failure } \\
\hline Yes & $0.94(0.90,0.99)$ & 0.018 & $0.961(0.919,1.004)$ & 0.077 \\
\hline No & $0.96(0.94,0.98)$ & $<0.001$ & $0.956(0.938,0.975)$ & $<0.001$ \\
\hline \multicolumn{5}{|l|}{ Diabetes mellitus } \\
\hline Yes & 0.95 (0.92, 0.98) & $<0.001$ & $0.94(0.91,0.97)$ & $<0.001$ \\
\hline No & $0.98(0.96,1.00)$ & 0.111 & $0.97(0.95,0.99)$ & 0.007 \\
\hline \multicolumn{5}{|l|}{ Hypertension } \\
\hline Yes & $0.96(0.94,0.99)$ & 0.005 & $0.95(0.92,0.97)$ & $<0.001$ \\
\hline No & $0.95(0.92,0.97)$ & $<0.001$ & $0.96(0.93,0.98)$ & $<0.001$ \\
\hline \multicolumn{5}{|l|}{ AKIN stage } \\
\hline 2 & $0.97(0.93,1.00)$ & 0.081 & $0.97(0.93,1.00)$ & 0.062 \\
\hline 3 & $0.95(0.93,0.97)$ & $<0.001$ & $0.95(0.93,0.97)$ & $<0.001$ \\
\hline \multicolumn{5}{|c|}{ Mechanical ventilation } \\
\hline Yes & $0.97(0.95,0.99)$ & 0.001 & $0.96(0.94,0.98)$ & $<0.001$ \\
\hline No & $0.95(0.91,1.00)$ & 0.036 & $0.95(0.91,0.99)$ & 0.027 \\
\hline \multicolumn{5}{|l|}{ SOFA score } \\
\hline$<8$ & $0.85(0.74,0.98)$ & 0.026 & $0.86(0.77,0.96)$ & 0.007 \\
\hline $8 \leq$ and $<12$ & $0.95(0.92,0.99)$ & 0.006 & $0.95(0.92,0.98)$ & 0.001 \\
\hline $12 \leq$ & $0.97(0.94,0.99)$ & 0.011 & $0.97(0.95,0.99)$ & 0.012 \\
\hline \multicolumn{5}{|l|}{ AKI causes } \\
\hline Sepsis & $0.96(0.94,0.98)$ & $<0.001$ & $0.95(0.93,0.97)$ & $<0.001$ \\
\hline Non-sepsis & $0.94(0.90,0.98)$ & 0.004 & $0.95(0.91,0.98)$ & 0.004 \\
\hline \multicolumn{5}{|l|}{ CRRT causes } \\
\hline Volume overload & $0.89(0.84,0.94)$ & $<0.001$ & $0.91(0.86,0.95)$ & $<0.001$ \\
\hline Metabolic acidosis & 0.95 (0.91, 0.99) & 0.015 & $0.94(0.90,0.98)$ & 0.002 \\
\hline Hyperkalemia & $1.01(0.86,1.17)$ & 0.935 & $1.03(0.91,1.18)$ & 0.617 \\
\hline Uremia & $1.02(0.92,1.13)$ & 0.667 & $0.97(0.88,1.07)$ & 0.549 \\
\hline Oliguria & $1.00(0.97,1.05)$ & 0.820 & $0.98(0.95,1.02)$ & 0.292 \\
\hline Other & $0.94(0.91,0.98)$ & 0.005 & $0.96(0.92,0.99)$ & 0.022 \\
\hline
\end{tabular}

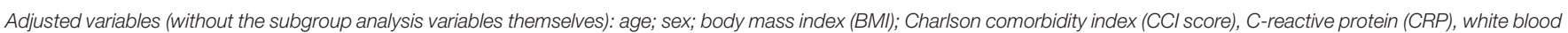

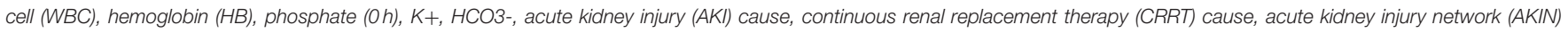
stages, CRRT does, 2 h urine output before CRRT initiation, sequential organ failure assessment (SOFA) score. 
was also found that the higher the ALB of patients, the higher the removal efficiency of serum phosphorus (as shown in Table 4).

\section{The Relationship Among ALB and 28-, 90-day Mortality, and the Removal Efficiency of Serum Phosphorus Explored by Curve Fitting Analysis}

In this study, we discovered that the higher the ALB, the lower the 28- and 90-day mortality of patients with AKI treated with CRRT, and the higher the delta phosphate in the curve fitting analysis. The following variables were adjusted in curve fitting analysis: age, sex, BMI, CCI, CRP, WBC, HB, phosphate $(0 \mathrm{~h})$ (except for delta phosphate), $\mathrm{k}^{+}, \mathrm{HCO}^{3-}$, AKI cause, CRRT cause, AKIN stages, CRRT dose, $2 \mathrm{~h}$ urine output before CRRT, and SOFA score (as shown in Figures 2-4).

\section{DISCUSSION}

According to this study, the higher the ALB before CRRT, the lower the 28- and 90-day mortality of patients with AKI treated with CRRT. It was also discovered that the higher the ALB before CRRT, the greater the serum phosphorus clearance efficiency.
Hypoalbuminemia had been demonstrated in certain studies to exacerbate AKI and to deteriorate the prognosis of individuals with AKI. According to Michael Joannidis's study, hypoalbuminemia accelerated the progression of AKI, increased the need for CRRT therapy for AKI patients, and was a significant predictor of AKI incidence (OR: 2.96, 95\% CI: 2.05-4.26) and mortality (OR: 2.47, 95\% CI: 1.51-4.05) (16). A retrospective cohort study of 381 critically ill patients by Praveen Kolumam Parameswaran also found that serum hypoproteinemia was an independent risk factor for AKI (OR: 1.810, 95\% CI: 1.1022.992) in critically ill patients (17). It also promoted the progress of AKI to CKD (17). In addition, we constructed a prediction model about AKI, and they found that ALB was an independent predictor of 28-day mortality of AKI patients (18). The possible mechanisms are as follows: (1) Plasma colloid osmotic pressure was critical in controlling the exchange of water between the inside and outside of blood vessels and in maintaining blood volume, while albumin was the primary molecule responsible for sustaining plasma colloid osmotic pressure; (2) Hypoalbuminemia, when accompanied by blood volume reduction causes the liquid in blood vessels to leak out, further reducing the volume of blood vessels and aggravating renal perfusion, along with worsening acute kidney damage ( 7 , $8,18-20)$.

TABLE 4 | Multivariate logistic regression analysis for delta phosphate.

\begin{tabular}{|c|c|c|c|c|}
\hline Exposure & delta phosphate (Unadjusted OR $95 \% \mathrm{Cl}$ ) & $P$-value & delta phosphate (Adjusted OR $95 \% \mathrm{Cl}$ ) & $P$-value \\
\hline Alb, g/L & $-0.04(-0.07,-0.01)$ & 0.004 & $-0.04(-0.07,-0.01)$ & 0.003 \\
\hline$<25$ & Reference & & Reference & \\
\hline $25 \leq$ and $<30$ & $-0.27(-0.60,0.06)$ & 0.111 & $-0.24(-0.58,0.09)$ & 0.153 \\
\hline Alb, g/L & $-0.04(-0.07,-0.01)$ & 0.004 & $-0.05(-0.08,-0.01)$ & 0.006 \\
\hline \multicolumn{5}{|l|}{ Alb, g/L } \\
\hline$<25$ & Reference & & Reference & \\
\hline $25 \leq$ and $<30$ & $-0.27(-0.60,0.06)$ & 0.111 & $-0.33(-0.76,0.10)$ & 0.137 \\
\hline $30 \leq$ & $-0.44(-0.81,-0.07)$ & 0.019 & $-0.61(-1.09,-0.13)$ & 0.013 \\
\hline $25 \leq$ and $<30$ & $-0.27(-0.60,0.06)$ & 0.111 & $-0.24(-0.68,0.19)$ & 0.271 \\
\hline $30 \leq$ & $-0.44(-0.81,-0.07)$ & 0.019 & $-0.60(-1.08,-0.11)$ & 0.016 \\
\hline \multicolumn{5}{|l|}{ Model 4} \\
\hline Alb, g/L & $-0.04(-0.07,-0.01)$ & 0.004 & $-0.04(-0.08,-0.01)$ & 0.014 \\
\hline \multicolumn{5}{|l|}{ Alb, g/L } \\
\hline$<25$ & Reference & & Reference & \\
\hline $25 \leq$ and $<30$ & $0.27(-0.60,0.06)$ & 0.111 & $-0.23(-0.67,0.20)$ & 0.293 \\
\hline $30 \leq$ & $-0.44(-0.81,-0.07)$ & 0.019 & $-0.58(-1.06,-0.09)$ & 0.020 \\
\hline
\end{tabular}

Model 1: Adjusted for age; sex; body mass index (BMI); Charlson comorbidity index (CCl score). Model 2: Adjusted for model 1 plus C-reactive protein (CRP), white blood cell (WBC), hemoglobin (HB), K+, HCO3-. Model 3: Adjusted for model 2 plus acute kidney injury (AKI) cause, continuous renal replacement therapy (CRRT) cause, acute kidney injury network (AKIN) stages, CRRT does, $2 \mathrm{~h}$ urine output before CRRT initiation. Model 4: Adjusted for model 3 plus sequential organ failure assessment (SO-O+FA) score. 


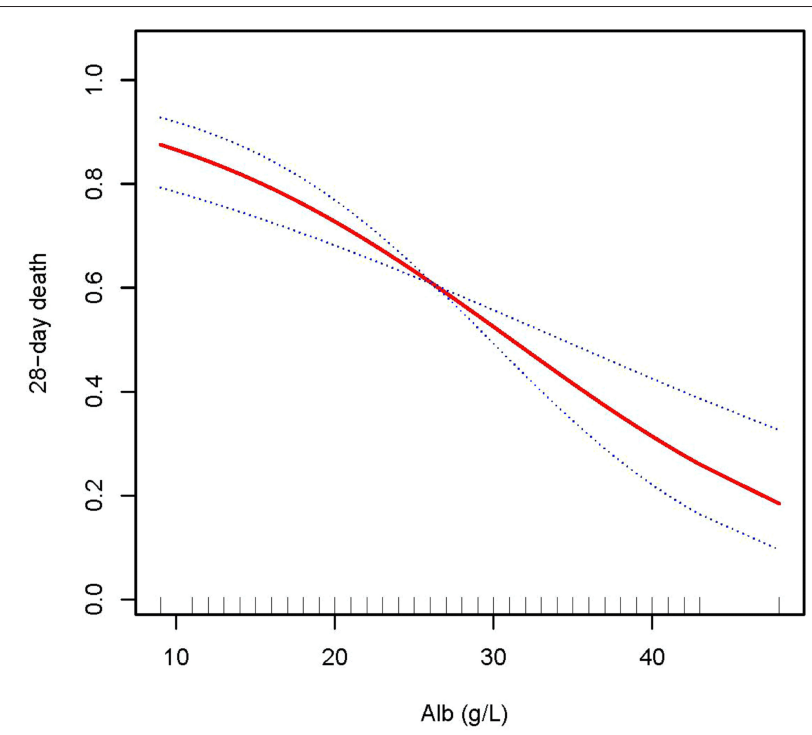

FIGURE 2 | Adjusted smoothing function of ALB for 28-day mortality. After adjusting for age, gender, body mass index (BMI), Charlson comorbidity index (CCl score), C-reactive protein (CRP), white blood cell (WBC), hemoglobin $(\mathrm{HB})$, phosphate $(\mathrm{O} \mathrm{h}), \mathrm{K}^{+}, \mathrm{HCO}^{3-}$, acute kidney injury (AKI) cause, continuous renal replacement therapy (CRRT) cause, (acute kidney injury network (AKIN) stages, CRRT dose, $2 \mathrm{~h}$ urine output before CRRT initiation, and sequential organ failure assessment (SOFA) score, curve fitting analysis showed that the 28-day mortality rate decreased as the ALB increased.

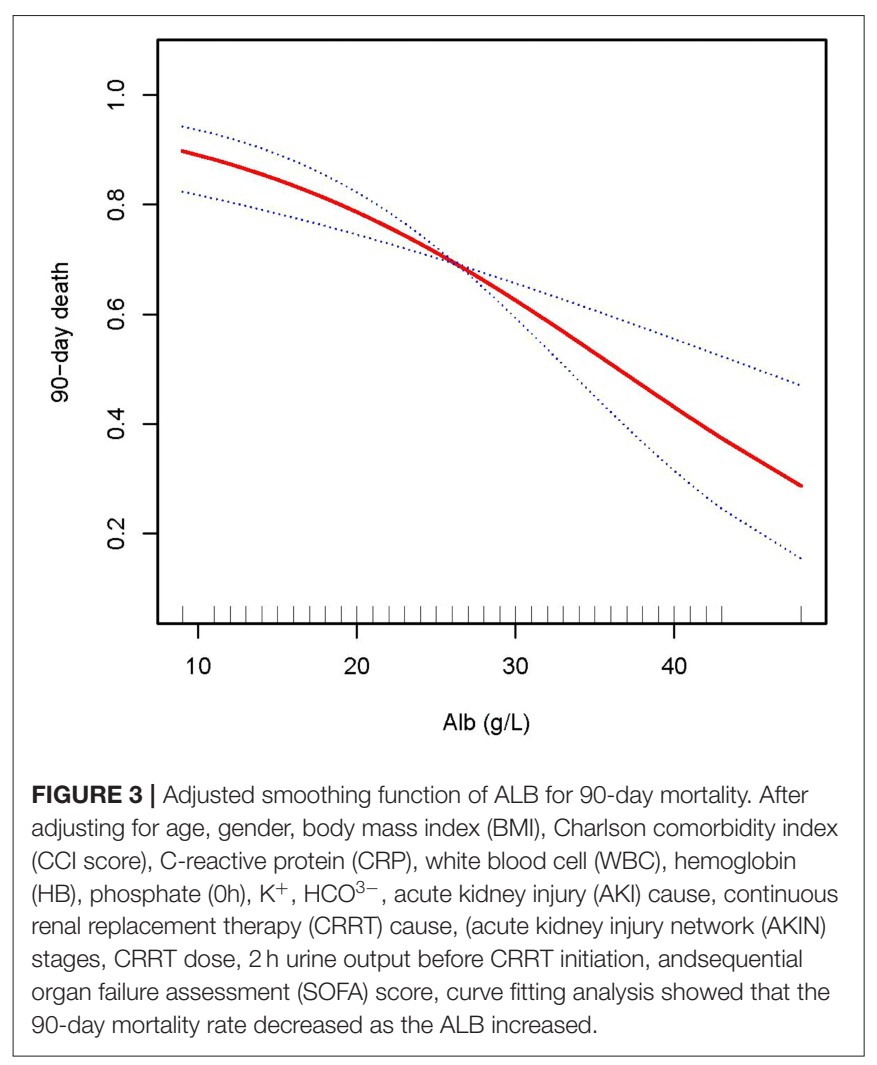

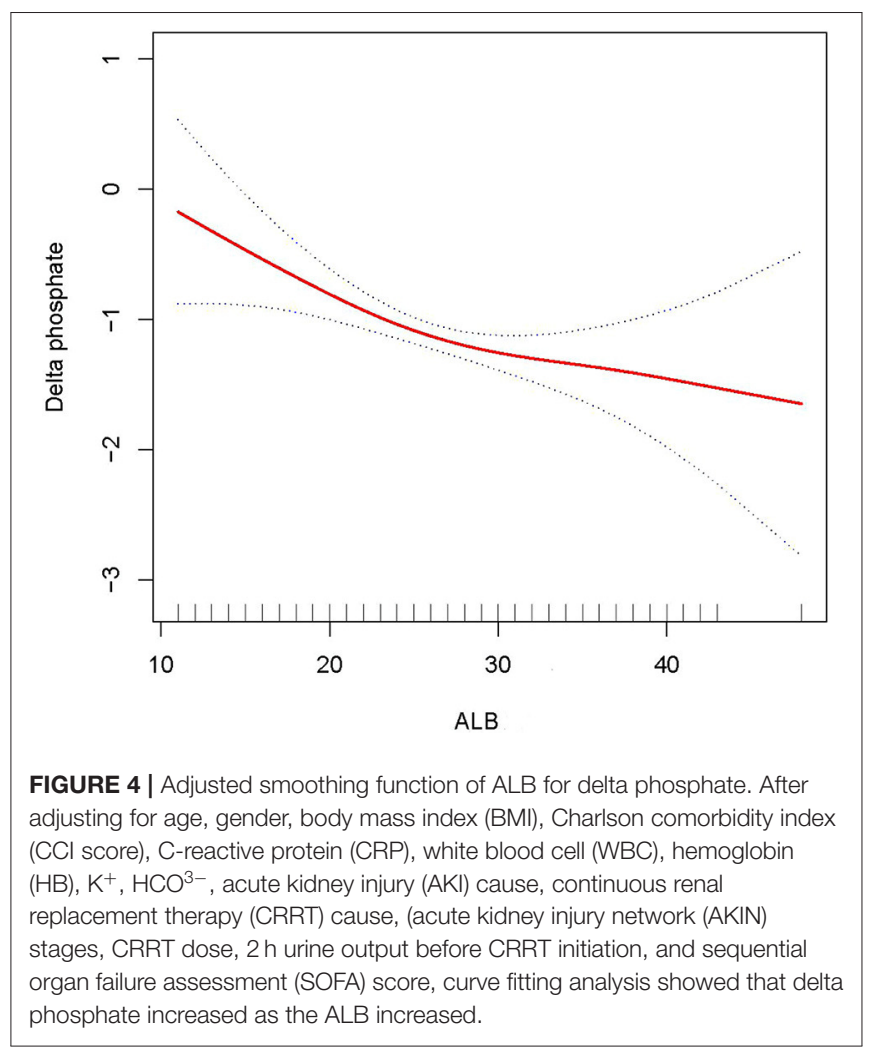

Continuous renal replacement therapy is often utilized in renal replacement therapy for critically ill patients, especially for those with hemodynamic instability (21). CRRT treatment for critically ill patients removes excess water and certain potentially toxic macromolecular compounds (22). Several studies had shown that insufficient CRRT treatment would result in adverse outcomes for patients. Simultaneously, it raised the risk of reCRRT therapy, thus increasing the medical risk, treatment cost, and length of stay (23). According to studies, serum phosphorus clearance in CRRT was positively associated with and reasonably near to the serum creatinine and urea nitrogen clearance in CRRT (4-6). Meanwhile, investigations had indicated that phosphate $(0 \mathrm{~h})$, phosphate $(24 \mathrm{~h})$, and delta phosphate were all linked to a higher risk of death in critically ill patients with septic AKI undergoing CRRT $(7,8,18,19)$. This study found that the higher serum ALB, the more serum phosphorus decreased after CRRT. However, since there were no indications of creatinine or urea nitrogen following CRRT therapy in this research, the connection between albumin and the clearance efficiency of CRRT treatment was not yet established and needed to be further investigated and validated.

Hypotension often occurs during the early stages of CRRT. The primary cause of hypotension in the early stages of CRRT is insufficient blood vessel content (24). Serum ALB is required to maintain enough blood vessel content. The higher the serum ALB, the more abundant the blood vessel content, so the lower the risk of hypotension during CRRT. According to studies, 
hypotension $1 \mathrm{~h}$ after the start of CRRT increased the hospital mortality and was an independent predictor of hospital mortality (25). To summarize, it was considered that the higher serum ALB might enhance the prognosis of critically ill patients with AKI and treated with CRRT: (1) The higher serum ALB, the higher the clearance efficiency of serum phosphorus; (2) The higher the serum ALB, the more the blood vessel content, and the lower risk of hypotension during CRRT.

Age, MAP, myocardial infarction, congestive heart failure, diabetes mellitus, hypertension, AKIN stage, mechanical ventilation, SOFA score, AKI causes, and CRRT causes were all subjected to the sensitivity analysis. It was found that ALB was associated with the 28-day mortality, except for patients with myocardial infarction, AKIN stage 2, and CRRT cause (hyperkalemia, uremia, and oliguria). The potential explanation was that the risk of mortality from myocardial infarction was very high, masking the effect of serum ALB; several studies indicated that patients with AKIN stage 2 who had CRRT could not improve their prognosis $(1,26)$, which may be the reason why serum ALB did not affect the prognosis of patients with myocardial infarction and AKIN stage 2 undergoing CRRT in this study. Studies had also shown that hyperkalemia, oliguria, or anuria were not related to patient mortality, which was similar to the findings of this research $(27,28)$.

\section{Strength of the Study}

(1) Through multivariate analysis, sensitivity analysis, and adjusting the potential confounding factors, this study got a more consistent conclusion: The higher the serum ALB, the better the prognosis of patients with AKI and treated with CRRT; (2) This study also made it clear that increasing the serum ALB might improve the clearance efficiency of serum phosphorus, and then improve the prognosis of critically ill patients with AKI and treated with CRRT.

\section{Limitations of the Study}

(1) This research belongs to a bi-center, retrospective, and observational cohort study and lacked important data such as length of the sessions, how many sessions throughout the ICU stay, and methods for coagulation. This lead to a certain possible danger of bias in this research, so its conclusion needed to be verified by a prospective study. (2) The AKIN standard was used to diagnose acute renal injury in this research; however, Kidney Disease Improving Global Outcomes (KDIGO) may be a superior AKI diagnostic standard. (3) Due to the lack of data on creatinine and urea nitrogen after CRRT in this study, only the relationship between ALB and serum phosphorus clearance was obtained in this study, while the relationship between ALB and CRRT clearance efficiency was needed to be further studied. (4) Albumin will rely on the condition of extracellular hydration and blood volume, although hydration quantity, nutritional status of the patients, and exogenous protein supplements might influence albumin measurements, and lead to a certain potential risk of bias. (5) In addition, some patients lost to follow-up, which increased the possibility of bias in the results.

\section{CONCLUSION}

The higher the serum ALB before CRRT, the lower the mortality of critically ill patients with AKI and treated with CRRT, and the higher the clearance efficiency of serum phosphorus.

\section{DATA AVAILABILITY STATEMENT}

The datasets presented in this study can be found in online repositories. The names of the repository/repositories and accession number(s) can be found at: https://datadryad.org// resource/doi:10.5061/dryad.6v0j9.

\section{ETHICS STATEMENT}

Agreement to participate in the study was not required because our review was a retrospective study of data reuse, and the patients' data was anonymous. Ethical approval was not provided for this study on human participants. The original author had obtained ethical approval when conducting his study. The ethics committee waived the requirement of written informed consent for participation in this study.

\section{AUTHOR CONTRIBUTIONS}

JL and HW participated in the research design, the revision of the manuscript, and data analysis. BS participated in the data analysis and writing of the paper. YG and $\mathrm{ZZ}$ participated in improving and revising the paper. HP provided substantial advice in designing the study and assisting in the division of labor, writing, and revising the paper. All authors contributed to the article and approved the submitted version.

\section{FUNDING}

This work was supported by Xi'an city "Science and Technology" + action plan, medical research project, Grant/Award Number: 2019115713XY012SF049.

\section{ACKNOWLEDGMENTS}

The authors thank Seung Hyeok Han for providing the data in the dryad database. 


\section{REFERENCES}

1. Doi K, Nishida O, Shigematsu $T$, Sadahiro $T$, Itami $N$, Iseki $K$, et al. The Japanese clinical practice guideline for acute kidney injury 2016. Clin Exp Nephrol. (2018) 22:985-1045. doi: 10.1007/s10157-0181600-4

2. Bagshaw SM, Wald R, Adhikari NKJ, Bellomo R, Da Costa BR, Dreyfuss $\mathrm{D}$, et al. Timing of initiation of renal-replacement therapy in acute kidney injury. N Engl J Med. (2020) 383:240-51. doi: 10.1056/NEJMoa200 0741

3. Karkar A, Ronco C. Prescription of CRRT: a pathway to optimize therapy. Ann Intensive Care. (2020) 10:32. doi: 10.1186/s13613-020-0648-y

4. Brunet S, Leblanc M, Geadah D, Parent D, Courteau S, Cardinal J. Diffusive and convective solute clearances during continuous renal replacement therapy at various dialysate and ultrafiltration flow rates. Am J Kidney Dis. (1999) 34:486-92. doi: 10.1016/S0272-6386(99)70076-4

5. Gong D, Ji D, Xie H, Xu B, Liu Y, Li L. The effects of dialysate and ultrafiltration flow rate on solute clearance during continuous renal replacement therapy. Zhonghua Nei Ke Za Zhi. (2001) 40:183-6.

6. Ratanarat R, Brendolan A, Volker G, Bonello M, Salvatori G, Andrikos E, et al. Phosphate kinetics during different dialysis modalities. Blood Purif. (2005) 23:83-90. doi: 10.1159/000082016

7. Jung S-Y, Kwon J, Park S, Jhee JH, Yun H-R, Kim H, et al. Phosphate is a potential biomarker of disease severity and predicts adverse outcomes in acute kidney injury patients undergoing continuous renal replacement therapy. PLoS ONE. (2018) 13:e0191290. doi: 10.1371/journal.pone.0191290

8. Wang H, Bai Z-H, Lv J-H, Sun J-L, Shi Y, Zhang Z-L, et al. The relationship and threshold of serum phosphate with regard to the 28-day mortality risk in sepsis patients undergoing continuous renal replacement therapy. J Int Med Res. (2020) 48:300060519831896. doi: 10.1177/0300060519831896

9. Kianfar E. Protein nanoparticles in drug delivery: animal protein, plant proteins and protein cages, albumin nanoparticles. J Nanobiotechnology. (2021) 19:159. doi: 10.1186/s12951-021-00896-3

10. Kelly A, Levine MA. Hypocalcemia in the critically ill patient. J Intensive Care Med. (2013) 28:166-77. doi: 10.1177/0885066611411543

11. Orlanski-Meyer E, Aardoom M, Ricciuto A, Navon D, Carman N, Aloi M, et al. Predicting outcomes in pediatric ulcerative colitis for management optimization: systematic review and consensus statements from the pediatric inflammatory bowel disease-ahead program. Gastroenterology. (2021) 160:378-402.e22. doi: 10.1053/j.gastro.2020.07.066

12. Herrmann FR, Safran C, Levkoff SE, Minaker KL. Serum albumin level on admission as a predictor of death, length of stay, and readmission. Arch Intern Med. (1992) 152:125-130.

13. Finfer S, Bellomo R, McEvoy S, Lo SK, Myburgh J, Neal B, et al. Effect of baseline serum albumin concentration on outcome of resuscitation with albumin or saline in patients in intensive care units: analysis of data from the saline versus albumin fluid evaluation (SAFE) study. BMJ. (2006) 333:1044. doi: 10.1136/bmj.38985.398704.7C

14. Frenette AJ, Bouchard J, Bernier P, Charbonneau A, Nguyen LT, Rioux $\mathrm{J}-\mathrm{P}$, et al. Albumin administration is associated with acute kidney injury in cardiac surgery: a propensity score analysis. Crit Care. (2014) 18:602. doi: 10.1186/s13054-014-0602-1

15. Wiedermann CJ, Wiedermann W, Joannidis M. Causal relationship between hypoalbuminemia and acute kidney injury. World J Nephrol. (2017) 6:17687. doi: $10.5527 /$ wjn.v6.i4.176

16. Wiedermann CJ, Wiedermann W, Joannidis M. Hypoalbuminemia and acute kidney injury: a meta-analysis of observational clinical studies. Intensive Care Med. (2010) 36:1657-65. doi: 10.1007/s00134-010-1928-Z

17. Shao M, Wang S, Parameswaran PK. Hypoalbuminemia: a risk factor for acute kidney injury development and progression to chronic kidney disease in critically ill patients. Int Urol Nephrol. (2017) 49:295-302. doi: 10.1007/s11255-016-1453-2

18. Bai Z-H, Guo X-Q, Dong R, Lei N, Pei HH, Wang H. A nomogram to predict the 28-day mortality of critically ill patients with acute kidney injury and treated with continuous renal replacement therapy. Am J Med Sci. (2021) 361:607-15. doi: 10.1016/j.amjms.2020.11.028

19. Kee YK, Kim D, Kim S-J, Kang D-H, Choi KB, Oh HJ, et al. Factors associated with early mortality in critically ill patients following the initiation of continuous renal replacement therapy. J Clin Med. (2018) 7:100334. doi: 10.3390/jcm7100334

20. Peerapornratana S, Manrique-Caballero CL, Gómez H, Kellum JA. Acute kidney injury from sepsis: current concepts, epidemiology, pathophysiology, prevention and treatment. Kidney Int. (2019) 96:1083-99. doi: 10.1016/j.kint.2019.05.026

21. Tandukar S, Palevsky PM. Continuous renal replacement therapy: who, when, why, and how. Chest. (2019) 155:626-38. doi: 10.1016/j.chest.2018.09.004

22. Lobo VA. Renal replacement therapy in acute kidney injury: which mode and when? Indian J Crit Care Med. (2020) 24:S102-6. doi: 10.5005/jp-journals-10071-23383

23. Fayad AI, Buamscha DG, Ciapponi A. Intensity of continuous renal replacement therapy for acute kidney injury. Cochrane Database Syst Rev. (2016) 10:CD010613. doi: 10.1002/14651858.CD010613.pub2

24. Douvris A, Zeid K, Hiremath S, Bagshaw SM, Wald R, BeaubienSouligny W, et al. Mechanisms for hemodynamic instability related to renal replacement therapy: a narrative review. Intensive Care Med. (2019) 45:133346. doi: 10.1007/s00134-019-05707-w

25. Shawwa K, Kompotiatis P, Jentzer JC, Wiley BM, Williams AW, Dillon JJ, et al. Hypotension within one-hour from starting CRRT is associated with inhospital mortality. J Crit Care. (2019) 54:7-13. doi: 10.1016/j.jcrc.2019.07.004

26. Mandelbaum T, Scott DJ, Lee J, Mark RG, Malhotra A, Waikar SS, et al. Outcome of critically ill patients with acute kidney injury using the Acute Kidney Injury Network criteria. Crit Care Med. (2011) 39:265964. doi: 10.1097/CCM.0b013e3182281f1b

27. Bianchi S, Aucella F, Nicola L de, Genovesi S, Paoletti E, Regolisti G. Management of hyperkalemia in patients with kidney disease: a position paper endorsed by the Italian Society of Nephrology. J Nephrol. (2019) 32:499-516. doi: 10.1007/s40620-019-00617-y

28. Lee H-J, Son Y-J. Factors associated with in-hospital mortality after continuous renal replacement therapy for critically ill patients: a systematic review and meta-analysis. Int J Environ Res Public Health. (2020) 17:238781. doi: 10.3390/ijerph17238781

Conflict of Interest: The authors declare that the research was conducted in the absence of any commercial or financial relationships that could be construed as a potential conflict of interest.

Publisher's Note: All claims expressed in this article are solely those of the authors and do not necessarily represent those of their affiliated organizations, or those of the publisher, the editors and the reviewers. Any product that may be evaluated in this article, or claim that may be made by its manufacturer, is not guaranteed or endorsed by the publisher.

Copyright (c) 2021 Lv, Wang, Sun, Gao, Zhang and Pei. This is an open-access article distributed under the terms of the Creative Commons Attribution License (CC BY). The use, distribution or reproduction in other forums is permitted, provided the original author(s) and the copyright owner(s) are credited and that the original publication in this journal is cited, in accordance with accepted academic practice. No use, distribution or reproduction is permitted which does not comply with these terms. 